\title{
Utilización de la técnica radiográfica de Fitzgerald como método de diagnóstico en implantología oral y periodoncia
}

\section{The utilization of the Fitzgerald radiographic technique as a diagnostic method in implantology and periodontics}

\author{
Vázquez Diego J*, Errecaborde $M^{*}$, Estévez A*, Osende $N^{*}$, Ramírez MJ**, \\ Carvajal $E^{* * *}$
}

\section{RESUMEN}

Se presenta un trabajo en el que se pone de manifiesto la importancia de la técnica de Fitzgerald como método de diagnóstico en especialidades de la Odontología como Implantología oral y Periodoncia. Así mismo se pudo demostrar la mayor precisión en lo que respecta a tamaño y forma de las estructuras a radiografiar, comparándolo con otras técnicas intraorales y extraorales como la radiografía panorámica, las que magnifican la imagen resultante. Basado en lo expuesto se explica detalladamente la técnica intraoral de Fitzgerald y se realiza una revisión bibliográfica del tema.

Palabras clave: Técnica de Fitzgerald, implantología oral, periodoncia, radiología, isometría.

\section{SUMMARY}

We presented a work in which the importance of the Fitzgerald technique as a diagnostic method, both in oral implantology and periodontics, is emphasised. On the other hand, it could be demonstrated its higher precision regarding the size and shape of the oral structures to be radiographed, as compared with other intraoral and extraoral techniques, such as panoramic radiography, which magnify the resulting images. Based on the exposed herein, a detailed explanation of the Fitzgerald intraoral radiographic method is provided, after reviewing the literature on this subject.

Key words: Fitzgerald technique, oral implantology, periodontics, radiology, isometrics.

Fecha de recepción: 5 de noviembre de 2008.

Aceptado para publicación: 11 de noviembre de 2008.

* Jefe de Trabajos Prácticos Regular. Cátedra de Radiología. Facultad de Odontología. Universidad de Buenos Aires. Argentina.

** Ayudante de Primera Regular. Cátedra de Radiología. Facultad de Odontología. Universidad de Buenos Aires. Argentina.

*** Profesor Adjunto Regular. Cátedra de Radiología. Facultad de Odontología. Universidad de Buenos Aires. Argentina.

Vázquez Diego J, Errecaborde M, Estévez A, Osende N, Ramírez MJ, Carvajal E. Utilización de la técnica radiográfica de Fitzgerald como método de diagnóstico en implantología oral y periodoncia. Av. Odontoestomatol 2009; 25 (4): 203-208. 


\section{INTRODUCCIÓN}

La técnica radiográfica de Fitzgerald o también llamada del paralelismo o cilindro largo cuyo debe su nombre al Dr. F. Gordon Fitzgerald, quien la desarrolla con el objetivo de obtener registros correctos en cuanto a forma y tamaño. brindándonos la posibilidad de evitar superposiciones o adiciones de zonas anatómicas vecinas.

Esta técnica se basa en el hecho de lograr una proyección con la menor distorsión geométrica posible, cumpliendo los requisitos de una radio proyección ideal: el rayo central debe incidir de forma perpendicular al objeto y la película siendo éstos paralelos, pasando por el centro de la estructura de interés con una distancia mínima desde la salida anódica de la radiación al objeto a radiografiar de $40 \mathrm{~cm}$, el doble que las demás técnicas retroalveolares. De esta manera disminuye el ángulo de radio proyección obteniendo así una imagen isométrica e isomorfa. Dada la distancia empleada, el tiempo de exposición deberá aumentarse 4 veces debido a que la intensidad de la radiación es inversamente proporcional al cuadrado de la distancia según la fórmula $I=1 / \mathrm{d}^{2}$. Cabe destacar que la utilización de películas de alta velocidad como F o G, puede minimizarse esta proporción. La imagen resultante presentará entonces una menor distorsión geométrica respecto a la que se obtiene con la técnica de la bisectriz, por lo que debiera ser la técnica de elección. Una tercera condición técnica indica que el rayo central debe incidir a la altura de las crestas óseas interalveolares, es por eso que es un método ideal para el diagnóstico de las lesiones óseas en la enfermedad periodontal (1-3).

Sin embargo, para que los planos de la película y el objeto sean paralelos, es necesario, en algunas zonas anatómicas un sistema de sujeción de la película que permita su colocación paralela al diente. Con estos dispositivos especiales llamados sostenedores, se mantienen en idénticas relaciones de paralelismo y perpendicularidad la película, la región a radiografiar y el tubo de Rayos X, situación repetible en sucesivos exámenes comparativos (4-7).

El objetivo de este trabajo es demostrar la utilidad de la técnica de Fitzgerald como método de diagnóstico en implantología oral y periodoncia en comparación con otras técnicas radiográficas convencionales.

\section{MATERIALES Y MÉTODOS}

Se realizaron estudios radiográficos panorámicos con un equipo ortopantomógrafo de movimiento helicoidal de última generación marca Cranex Thome con valores utilizados de $65 \mathrm{Kv}, 10 \mathrm{~mA}$, tiempo de exposición: 17 segundos.

Las películas utilizadas para esta técnica es de 15 $\mathrm{cm}$ por $30 \mathrm{~cm}$. Marca Agfa y chasis rígido con pantallas reforzadoras sensible al espectro de luz verde.

Los estudios radiográficos intraorales se realizaron con un equipo radiográfico marca Dabi Atlante con valores fijos de $70 \mathrm{Kv}, 8 \mathrm{~mA}$ y tiempo de exposición promedio de 0,3 seg. por toma. Se utilizaron películas retroalveolares de $3 \mathrm{~cm}$ por $4 \mathrm{~cm}$. Marca Agfa.

Se utilizaron los elementos de protección según normas vigentes.

El tratamiento quirúrgico se llevó a cabo con material de antisepsia descartable, y para la confección de los campos. Anestesia local: 2 anestubos marca totalcaína, aguja de anestesia corta mango de bisturí $N^{\circ} 3$, hoja No $^{\circ}$; ; separadores de farabeuf, periostótomo, sindesmótomo, fresas quirúrgicas para la colocación del implante, instrumental rotatorio con su fisiodispenser. Implante 13 milímetros de longitud, hilo de sutura 0000, porta aguja, tijera, gasa estéril, solución fisiológica y solución desinfectante. Regla milimetrada.

\section{RESULTADOS}

\section{Caso 1}

Se realiza una toma radiográfica intraoral con la técnica de Fitzgerald (T.F.) a un paciente de sexo masculino de 39 años en el que se colocará un implante dentario para reponer el segundo premolar superior derecho, extraído 6 meses antes. Se observa en la imagen una distancia entre la cortical del seno maxilar y la cortical de la cresta de 16 milímetros (Fig. 1). 


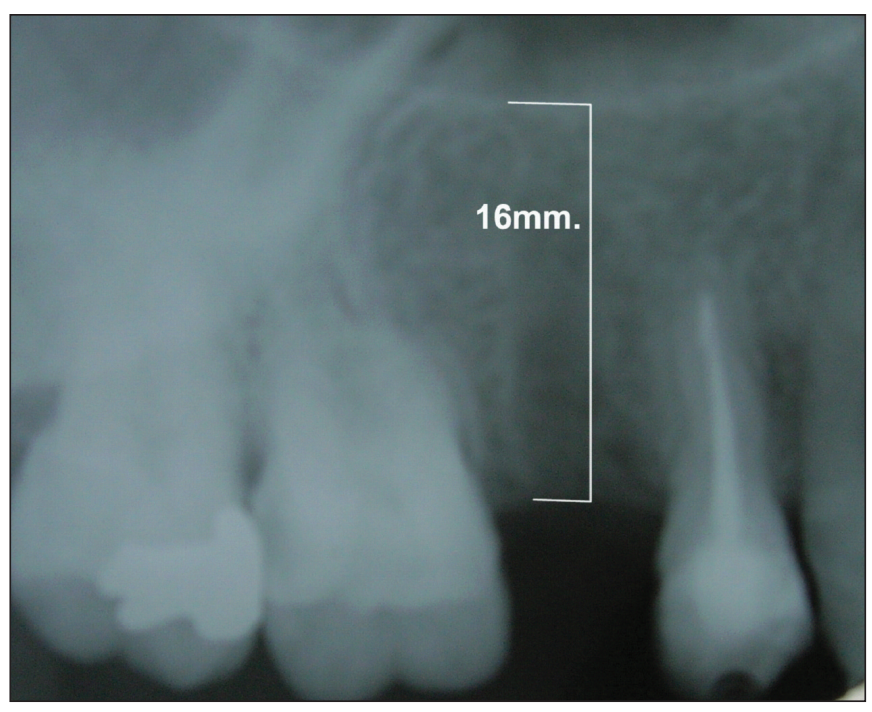

Fig. 1. Radiografía preoperatorio con la técnica de Fitzgerald.

Se realiza a posteriori una radiografía panorámica (T.P.), realizada la misma medición sobre los mismos reparos, se mide un aumento marcado de la distancia con respecto a la T.F que llega a los 23 milímetros $=43,75 \%$ más (Fig. 2).

Se proyecta entonces la cirugía para colocar un implante de 13 milímetros de longitud. Ya en el acto quirúrgico y una vez utilizada la fresa redonda y la

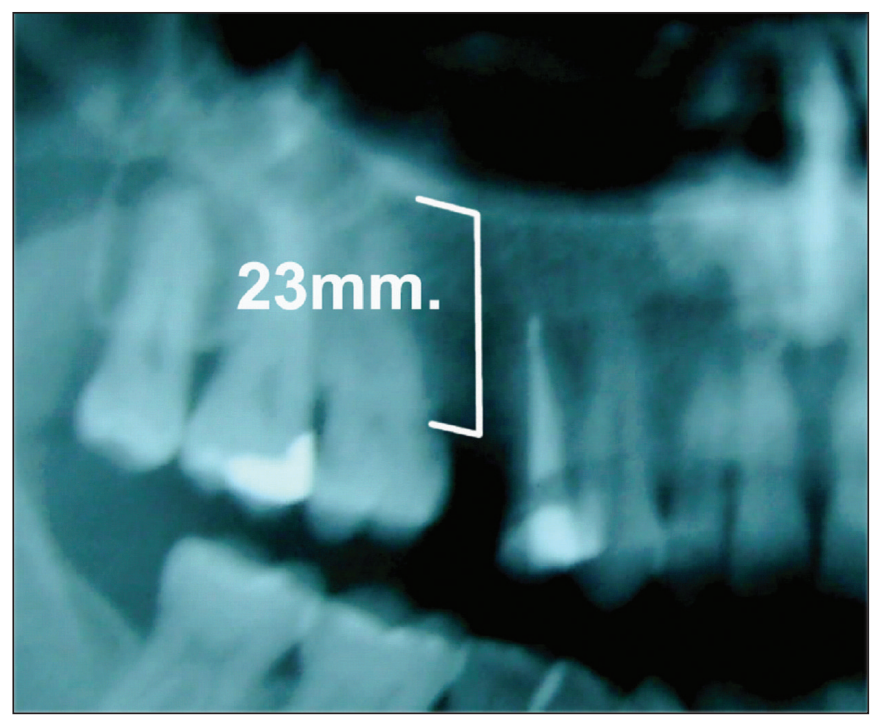

Fig. 2. Radiografía panorámica. fresa lanza, se coloca el pin paralelizador del que se introducen 5 milímetros. Se toma una radiografía con la técnica del paralelismo utilizando rollos de algodón estériles para paralelizar la película. Se mide nuevamente la distancia que hay entre las corticales del seno y de la cresta ósea y se detecta un leve aumento de la misma (17 milímetros) con respecto a la técnica preoperatorio. Bebido a la dificultad para poder realizar la toma intraoperatoria, se mide el pin paralelizador y se observa en la misma, un diámetro de 5,5 milímetros desde la cresta, lo cual nos da una mínima distorsión $=10 \%$, respecto a los 5 milímetros reales del pin introducido. Esto nos permite trabajar con un margen importante en lo que se refiere a la instrumentación a realizar en próximos pasos operatorios (Fig. 3).

En la figura 4 se realiza la toma radiográfica en el post operatorio inmediato en la que se constatan las mismas mediciones entre las corticales realizadas en la toma preoperatoria. La medición del implante en la radiografía nos reproduce con exactitud la medida real del mismo. La figura 5 nos muestra la misma imagen 6 meses posteriores a la cirugía, en la que se

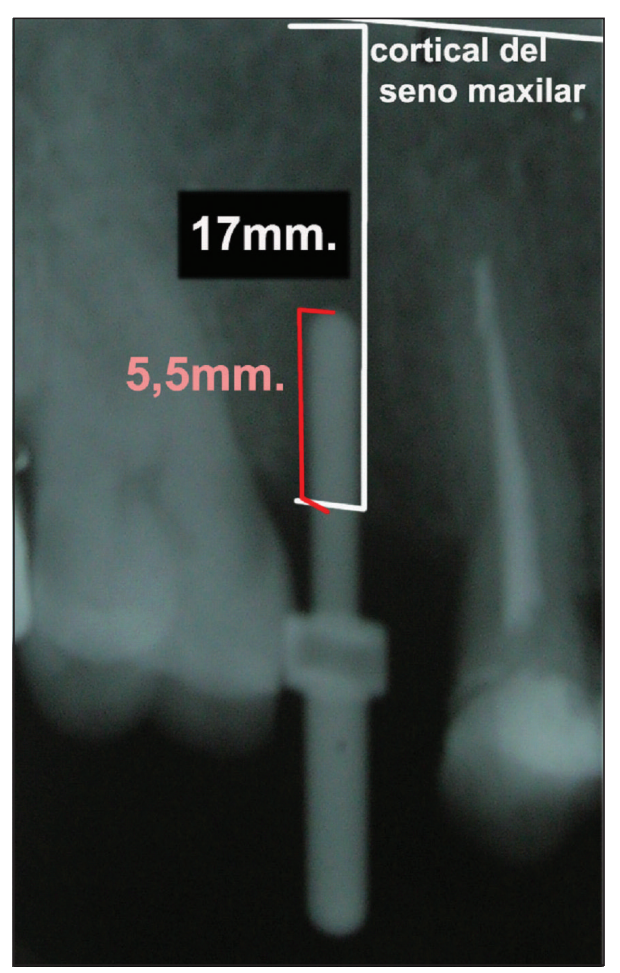

Fig. 3. Radiografía intraoperatoria. T.F. 
Fig. 4. Radiografía postoperatoria inmediata.
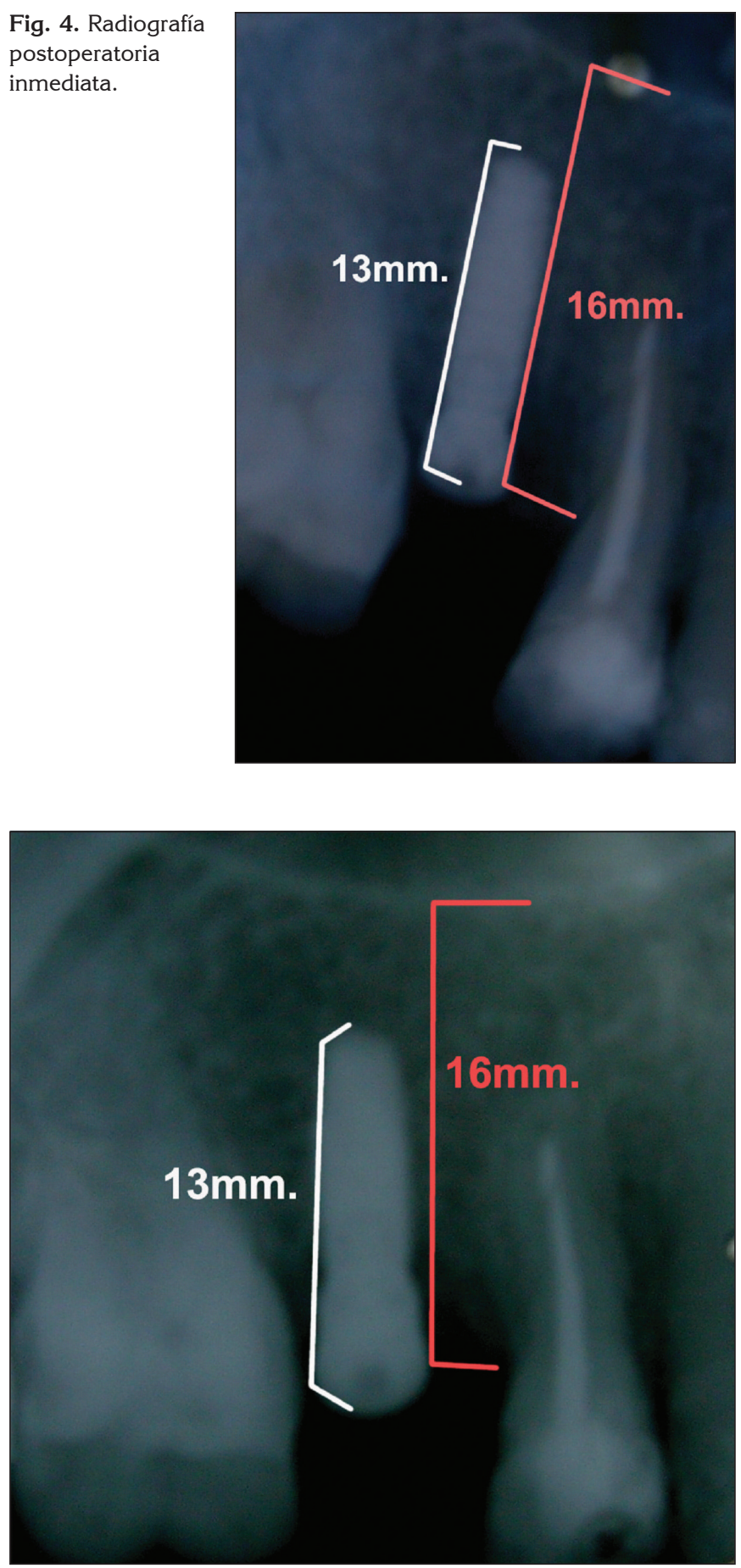

Fig. 5. Radiografía postoperatoria 6 meses después. Ambas, tomadas con la técnica de Fitzgerald.

reiteran las mediciones de la toma anterior con igual técnica radiográfica. Obsérvese una leve reabsorción de la cortical de la cresta y la oseointegración formada alrededor del implante.

\section{Caso 2}

Se realizan estudios radiográficos convencionales a un paciente de sexo femenino de 43 años de edad derivada por el especialista en periodoncia, para evaluar las crestas óseas interdentarias. Se hacen 2 tomas, la primera con la técnica de Dieck o cilindro corto (Fig. 6), en el mismo el rayo central incidió a nivel del ápice de las piezas dentarias en la bisectriz imaginaria formada entre el objeto a radiografiar y la película, con una distancia de $20 \mathrm{~cm}$. En la misma se observa la superposición de la apófisis piramidal del maxilar superior como indica la flecha produciendo un efecto de adición radiopaco con las piezas dentarias, se observa también una aparente imagen acortada de 1,3 milímetros entre el reborde óseo marginal y un punto de referencia dado por las cuñas adamantinas proximales del segundo premolar y el primer molar superior.

La figura 7 muestra una imagen realizada con la técnica de Fitzgerald, aumentando al doble la distancia foco-objeto respecto a la técnica anterior e incidiendo el rayo central a nivel de las crestas óseas interalveolares. Se observa con claridad la desproyección de la apófisis piramidal del maxilar superior y la distancia entre el reborde óseo marginal y el punto de referencia ya mencionado. En esta toma las cuñas adamantinas proximales del segundo premolar y el

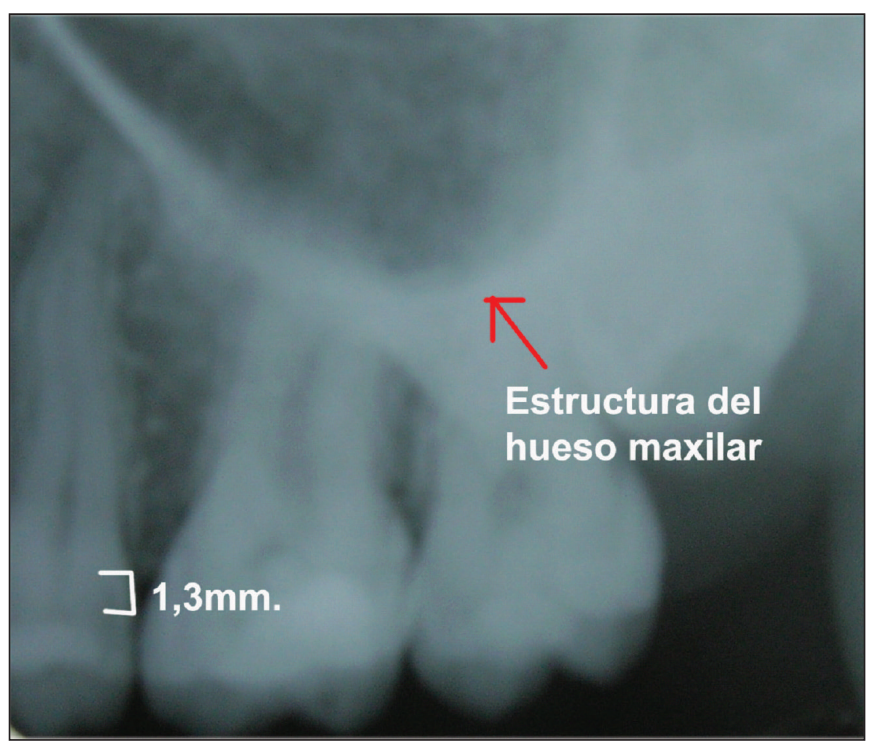

Fig. 6. Técnica del cilindro corto. 


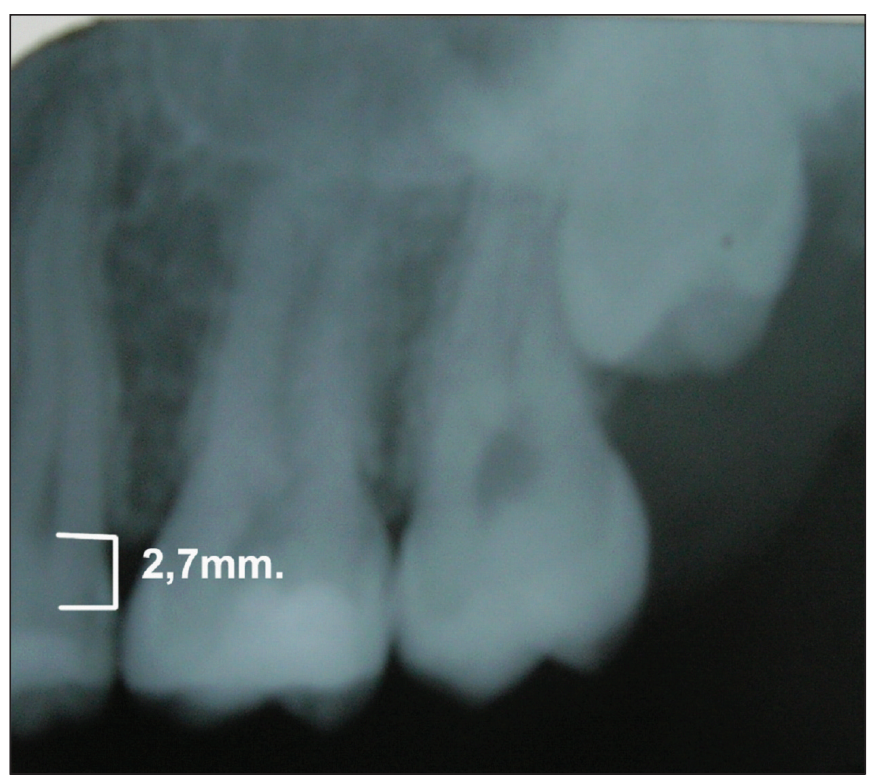

Fig. 7. Técnica del cilindro largo.

primer molar superior se ven aumentadas en 1,4 milímetros con respecto a la incidencia anterior $=$ $2,7 \mathrm{~mm}$ equivalente a un $100 \%$.

\section{DISCUSIÓN Y CONCLUSIÓN}

Se pudo establecer que la técnica de Fitgerald es el estudio radiográfico convencional aconsejable en implantología oral como primer método de diagnóstico preoperatorio, antes de realizar un estudio de alta complejidad, en el intraoperatorio y a posteriori del mismo. También en periodoncia es aconsejable ya que nos brinda la posibilidad de obtener imágenes más isométricas e isomorfas. Para explicar la obtención de imágenes mas similares en tamaño y forma con el objeto a radiografiar es necesario comparar la misma con una técnica en la que la distancia es menor (7-9).

En la figura 8 se observa un aumento del tamaño de la imagen debido a la cercanía del tubo radiográfico al objeto y, por ende un aumento del ángulo de radioproyección entre ambos que da como resultado una magnificación del objeto incidido.

En la figura 9 se observa como se aleja el tubo radiográfico, por ende se disminuye el ángulo de radiopro-

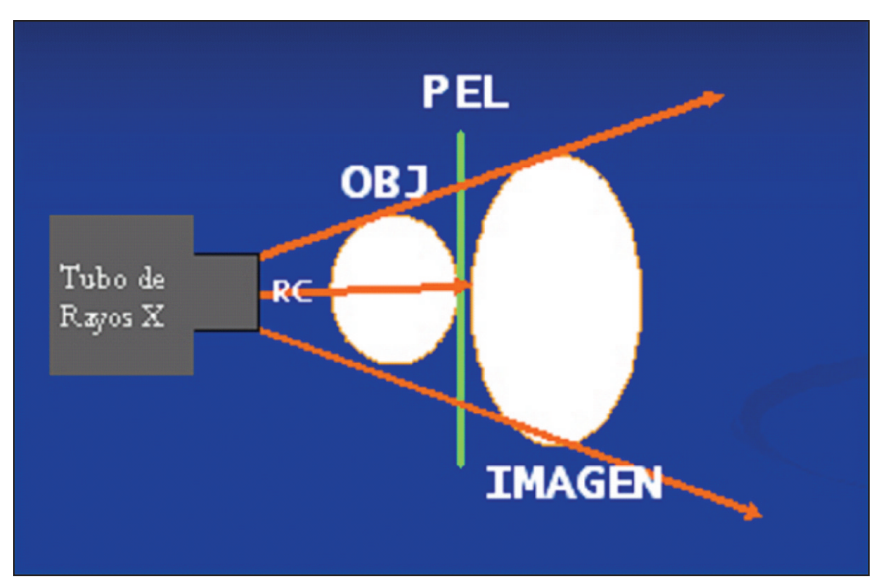

Fig. 8. Ángulo de radioproyección.

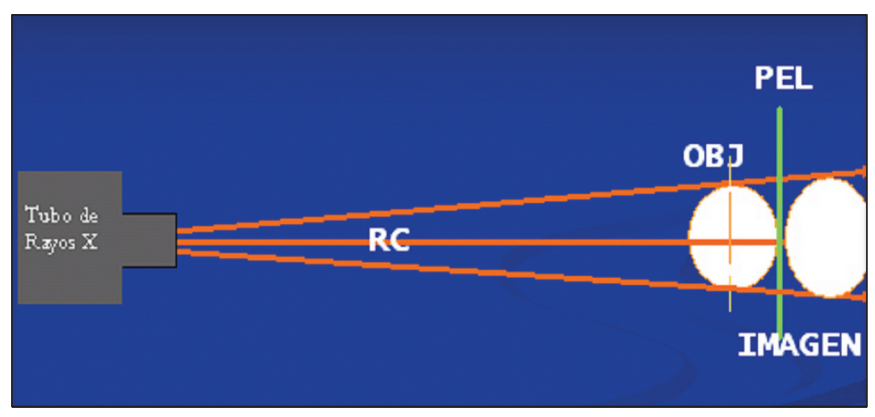

Fig. 9. Ángulo de radioproyección menor.

yección y nos da como resultado una imagen parecida en tamaño con respecto al objeto real (9-12).

El objetivo de este trabajo es poder demostrar la importancia de la técnica de Fitzgerald como método de diagnóstico en especialidades de la rama de la odontología como lo es la implantología oral y la periodoncia, para poder obtener mayor precisión en lo que respecta a tamaño y forma del objeto a radiografiar comparándolo con otras técnicas como la retroalveolar de Dieck o la extraorales como lo es la panorámica, en las que se manifiesta una apreciable magnificación de la imagen resultante.

\section{BIBLIOGRAFÍA}

1. Rushton, V.E. and Horner, K. The use of panoramic radiology in dental practice. J Dent 1996;24:185201. 
2. Espinoza, Norelkys. Equipos de alta tecnología en imagen digital y su apliación en la odontología. Acta odontol Venez 2005(ago);43(2).

3. Martínez, María Elisa; Diagnóstico por imágenes en implantología; Rev. Fundac. Juan José Carraro. 2003 (ago);8(17):33-7.

4. Rubira, Izabel Regina Fischer; Diagnóstico por imagem em periodontia; Tunes, Urbino da Rocha; Rapp, Gisela Estela. Atualizaçäo em periodontia e implantologia. Säo Paulo, Artes Médicas, 1999.

5. Campos Junior, Aguinaldo; Lourenço, Eduardo José Vegas; Taba Júnior, Mário; Aragones, Liane Cassol Argenta; Tunes, Urbino da Rocha; Rapp, Gisela Estela. Atualizaçäo em periodontia e implantologia. Säo Paulo, Artes Médicas, 1999.

6. Fonseca, Miguel Mario; Ferreyra, Fernando. Consideraciones biológicas, quirúrgicas y protésicas sobre el diagnóstico para la inserción de implantes oseointegrados. Rev Fac Odontol (Córdoba);21/22(1/2):69-86, ene. 1993-dic. 1994.

7. Application of digital radiography to implantology. J Dent Symp 1993 Aug;1:30-3
8. Digital radiology for implant treatment planning and evaluation. Dentomaxillofac Radiol 1992 Nov;21(4):203-7.

9. Goaz, PW y White, S. Radiología Oral. Principios e Interpretación. Editorial Mosby, 1995.

10. Haring J, Lind L. Radiología Dental. Editorial Mc Graw-Hill, Interamericana México, 1997.

11. Pasler F. Atlas de Radiología Odontológica. Editorial Masson-Salvat, 1992.

12. Hintze $H$, Wenzel A. Oral radiographic screening in Danish children. Scand J Dent Res 1990;98: 47-52.

\section{CORRESPONDENCIA}

J. Vázquez

Gdor. Marcelino Ulgarte $15481^{\circ}$ piso departamento "C" Olivos C P 1636

Provincia de Buenos Aires

Argentina

E-mail: jv983@hotmail.com.ar eecarbajal@yahoo.com.ar 